\title{
Analysis of Design Principles and Elements of Square Fitness Dance
}

\author{
Ailuan Huang \\ (Hainan Tropical Ocean University, Sanya, 572000, China)
}

Keywords: Square fitness dance; Design principles; Design elements

\begin{abstract}
This paper adopts the research methods of literature and investigation to analyze the design principles and elements of square fitness dance of China to enrich and improve the theoretical research system of that and to make the square fitness dance culture be more scientific and practical so that it can play a greater role in the national fitness cause.

At present, the major cities in China have entered the aging society, and the national fitness movement has become a common concern of the state and the masses. Because it is simple to learn, easy to participate and be in various forms, the square fitness dance quickly went popular all over the country. Today, it has developed into one of the main methods for all people to participate in physical exercises, and has become the first body building choice for the middle-aged and elderly people. As one of the sports body-building project, the square fitness dance not only enriches the amateur cultural life of broad masses of the people, but also creates a healthy and civilized lifestyle for them. At the same time, it can boost the development of national fitness campaign. However, many of the popular square dancing is not scientifically designed and problems still exist. The theory of the square fitness dance at home and aboard is also deficient. Therefore, the paper analyses the design principles and elements of square fitness dance to promote the square fitness dance in a healthy and scientific direction so that it can better serve the broad masses of the people and provide accurate and objective data as well as decision reference for the relevant sports departments.
\end{abstract}

\section{Analysis of Design Principles of Square Fitness Dance}

\section{Simple and Feasible Principle}

For most people, the purposes of participating in the square fitness dance are to improve the health and to gain entertainment. If the design is too complex and uncomfortable, the participants will have some difficulties to learn the dance or even cannot learn. They will lose the interest in continuing learning Therefore, to better promote the popularization of the routine actions of the square fitness dance, the actions we design must be simple to learn and easy to popularize so that participants can master the square fitness dance routines in a relatively short period and feel the fun of square fitness dance. In this way, the participants can practice the actions with ease, freedom, cheerfulness and a sense of success. Only in this way can the participants enjoy the happiness brought by the square fitness dance. The square fitness dance can help the participants to cultivate sentiments, relieve their physical and mental pressures and shape their bodies.

\section{Scientific and Reasonable Principle}

Scientific and rational arrangement of sports load is the basic condition to ensure that participants can achieve the desired goal. Only the appropriate exercise load can achieve good exercise effect in the physical exercise. If the exercise load is too small, the exercise effect is not ideal; if the exercise load is too large and that is beyond the physical and physiological capacity of the participants, it will cause some serious adverse effects. We usually monitor the exercise load by the index of heart rate in sports activities. The simplest method to measure the heart rate is to measure the pulse, for the pulse 
rate is consistent with the number of heartbeats for a normal person (except the patients with arrhythmia). The measurement of the pulse rate in one minute is usually used as the heart rate. The maximum heart rate is measured by the formula no matter at home and abroad, that is " 220 -the age= maximum heart rate". And reaching the $60 \%-80 \%$ of the maximum heart rate is the best. Within the above range, the higher the heart rate is, the better the exercise effect is. Therefore, the exercise load of the routine actions of the square fitness dance must be controlled in the optimal range to ensure the safety and effectiveness of the exercise.

\section{Safe and Effective Principle}

Under normal circumstance, the fitness dance it is rarely lead to the sports injuries or the occurrence probability of sports injury in the square fitness dance is far less than that in football, badminton, swimming and other sports. However, if you do not pay attention to choosing scientific and reasonable means, methods and contents, the fitness dance will also cause sports injury. For example, the repeated raising of the arm, repeated high impact actions during a short period or running, jumping and twisting for a long time will cause acute or chronic exercise injury.

Therefore, in the design process of square fitness dance works, we must select the effective actions basing on the safety of these actions. We usually do not choose these actions that with the characteristics of high effectiveness but unsafe. At the same time, the actions content should be designed suitable for the people of general physique or weak physique.

\section{Comprehensive Fitness Principle}

The final goal of physical exercise is the fully exercise of the head, neck, limbs, trunk and other parts of the body through the participation in the sports activities. Therefore, the content selection of the square fitness dance routines should include the actions of the head, neck, upper and lower limbs, trunk and other parts. The neck actions should include the actions of raising, bowing, turning left and turning right; the upper limb movements should include the movements of bending, stretching, pushing, raising, lifting, swinging and circling movements; the lower limb movements should include walking, jumping, squatting, stepping, running, knee up and kicking the legs; the trunk movements should include rotating, modeling and other various types of actions. At the same time, the movements should be designed according to the symmetry principle of the left and right sides. The movements should not be designed to exercise only the right or left side of the body, which will lead to a lack of harmonious and balanced development of the body. Secondly, the magnitude, direction and length of the movements should be designed in the direction of left and right, front and back, up and down, and the diagonal lines. In this way, people can improve their physical qualities comprehensively, enhance the cardiopulmonary and cardiovascular functions and meet the goal of participating in physical exercise through the learning of the square fitness dance.

\section{Analysis of Design Elements of Square Fitness Dance}

\section{Increase Artistry and Fun in the Actions}

With the increasing choices of the sports events, the requirements of the content, methods and forms of the physical exercise proposed by the mass are higher and higher. Therefore, in the design of the square dance exercise, we should do an ingenious design to add the artistry and fun in the routine actions and combine actions as much as possible in addition to considering the effect of the exercise. A set of beautiful and interesting routines can greatly enhance the learners' interest. We can select simple, fashionable, fluent and graceful movements, and make some innovative improvements in the content, methods, rhythms, ranges and routes of the actions. The design should avoid the movements that are too complex and old-fashioned; otherwise it is difficult and boring for the practitioners to learn. 


\section{Clarify Purpose and Task of the Design}

We must make the purpose and task of fitness dance development clear before designing any set of fitness dance. Different purposes and tasks determine different design routines. For example, if the routines are used in performance, the ornamental value will be its main objective. The movement, rhythm, body posture and expression must be as graceful and generous as possible. The actions should avoid unnecessary repetition and make full use of every space in the site. If the main design purpose is to exercise, then the action content, the exercise form, method, load, intensity and magnitude should be designed to improve the physique and health. We can appropriately increase the range of motion, strength, or improve the upper limb movements to exceed the horizontal level of the heart in the design so as to enhance the heart rate of the exerciser. We can improve the cardiopulmonary function and the cardiovascular function in the movement process only in the condition that the heart rate achieves a certain target range. In this way, participants can achieve physical fitness and improve physiological function through the square dance.

\section{The Actions should be Designed to Contain Local National Elements}

Different nationalities in our country have different national cultural symbols, personalities, action styles and expression forms. For example, "Sandaowan" of Li nationality is the most unique and basic movement of the action style and expression form of Li nationality. It is incisive and vivid in the traditional dance of $\mathrm{Li}$ nationality. Therefore, in the actions design of the square fitness dance, in addition to paying attention of the diversity and pluralism of the actions, we must extract the most typical or representative action elements and integrate them into the design of square fitness dance routines. Only in this way, can we make the design of the routine movements be fashionable and have local national characteristics; only in this way, can we arouse the audience's sympathy and refreshing feeling and inherit the local national culture.

\section{The Actions should be Designed to be Naturally Fluent and Orderly Coordinated}

According to the physiological rules of human movement and the requirements of human physiological anatomy, the collocation and cohesion of the whole set of movements should be scientific, rational and natural. The combination or integration of the different types of actions or the sudden changes of the movement gravity center may lead to the difficulty for the participants to master the actions. It is particularly difficult to keep up with the teaching for the beginners. For example, in the condition that the learner uses a cross over step of the right foot from the first beat to the fourth beat, if the design of the fifth beat to eighth beat is a cross over step of the left foot, the cohesion of actions is very smooth and reasonable. But, if the design of the fifth beat to eighth beat is also a cross over step of the right foot, it is not very easy to keep the movements for a beginner. The beginner has to stop. At the same time, the kind of design can easily make the people with poor balance injured. Therefore, in the process of designing the whole set of square dances, we should not only take the movements collocation into consideration, but also notice the connection among the movements. We should make sure that every action can be integrated scientifically, reasonably, naturally, fluently and effectively, and make sure that people can keep complete routine actions in mind as much as possible. At the same time, we must also follow the body characteristics and design the actions step by step according to the sequence of the single action, the combination action and the routine action. In this way, the routine actions of the designed square dance can be natural, smooth, coordinated, safe, and reliable and has a strong attraction.

\section{The Music should be Designed Dulcet and Perfectly Integrated into the Actions}

The design of the fitness dance or ordinary dance cannot do without the element of music. Music is not merely a kind of acoustics effect but expresses a kind of artistic conception and emotion. The good musical effect can not only stimulate people's imagination and thinking but also arouse people's ideological resonance and create good mood. If the music of a square fitness dance is very unpleasant or cannot express the meaning of the action, it will not be appreciated by the viewers and the practitioners. The action will become artistic, ornamental and vital only in the condition that the 
music is melodious and harmonious with the actions; otherwise, the actions will be monotonous and boring. Therefore, the music design of the square fitness dance must be melodious and the rhythm must be bright. It should be consistent with the actions' content, rhythm, emotion, intensity, style, range, fluctuation and change. Only the organic combination of the music and action is achieved can the real charm of the actions be expressed. As a result, people can easily stimulate the exercise passion and learning interest and relieve their exercise fatigue.

\section{Conclusions}

The square fitness dance is neither a world Olympic event nor an event of domestic championship, but it has a strong mass base and is booming in our country. This paper puts forward some suggestions for the design principles and elements of square fitness dance in our country, and hopes to provide theoretical and practical references for the development of the national fitness campaign in China.

\section{References}

[1] Peng Daoxiu. Current Situation of Square Fitness Dance in the Urban Areas of Hubei Province and Its Development Tendency [J]. Journal of Yangtze University (Natural Science Edition), 2016, 13(13): 62-65.

[2] Kuang Liehui. Study on the Development of Square Fitness Dance from Visual Angle of Harmony [J]. Journal of Beijing Sport University, 2014, 37(4): 32-37.

[3] Cao Jiwei, Yuan Yuan. A Study on Dilemma and Path of the Development of Square Dance in the Background of the Nation-wide National Fitness Strategy [J]. Bulletin of Sport Science \& Technology, 2017, 25(2): 99-100+125.

[4] Yao Xia. On the Composition of Mongolians Square Fitness Dance [J]. Wushu Studies, 2016, 1(12): 97-100+103. 